\title{
Desenvolvimento de um sabonete líquido a partir do extrato da casca do fruto da pitomba (Talisia esculenta)
}

\author{
Development of a liquid soap from the pitomba fruit peel extract (Talisia esculenta) \\ Desarrollo de un jabón líquido a partir del extracto de cáscara de frutas de pitomba (Talisia \\ esculenta)
}

\section{Resumo}

A Talisia esculenta (A.ST.-HIL.) Radlk, pertencente à família Sapindaceae e é muito consumida in natura principalmente no Nordeste do Brasil. O presente estudo teve como objetivo desenvolver um sabonete líquido com extrato da casca do fruto da pitomba que é um resíduo sólido que geralmente é descartado sem uso. As cascas foram trituradas e armazenadas em frascos de plástico fechado em congelador até o uso. Posteriormente, procedeu-se com preparo dos extratos hidroalcoólico e aquoso. Após esse processo, os extratos foram analisados quanto ao perfil fitoquímico (saponinas, fenóis e taninos e flavonóis, flavonas, flavononóis e xantonas). Em seguida, desenvolveu-se o sabonete líquido. Logo após realizou-se análise dos parâmetros organolépticos, pH e densidade aparente. Verificou-se que o sabonete apresentou discreta diferença no aspecto visual em temperatura ambiente e geladeira e cor em temperatura ambiente, podendo ser associado à presença de compostos bioativos. $\mathrm{O}$ pH foi 5,44 $\pm 0,13$ e a densidade $1,031 \pm 0,002 \mathrm{~g} / \mathrm{cm} 3$, de acordo com os valores reportados na literatura. O sabonete líquido desenvolvido apresentou características organolépticas, $\mathrm{pH}$ e densidade aparente adequados. Ademais, pesquisas posteriores deverão ser feitas para melhorias do produto e análises de eficácia.

Palavras-chave: Agroindústria; Bioativos; Extratos; Resíduos; Sapindaceae.

\section{Abstract}

Talisia esculenta (A.ST.-HIL.) Radlk, which belongs to the Sapindaceae family and is widely consumed in natura, mainly in Northeastern Brazil. The present study aimed to develop a liquid soap with extract from the pitomba fruit 
rind, which is a solid residue that is usually discarded without use. The husks were crushed and stored in closed plastic bottles in a freezer until use. Subsequently, the hydroalcoholic and aqueous extracts were prepared. After this process, the extracts were analyzed for their phytochemical profile (saponins, phenols and tannins and flavonols, flavones, flavononols and xanthones). Next, liquid soap was developed. Soon after, the organoleptic parameters, $\mathrm{pH}$ and bulk density were analyzed. It was found that the soap showed a slight difference in the visual appearance at room temperature and refrigerator and color at room temperature, which may be associated with the presence of bioactive compounds. The $\mathrm{pH}$ was $5.44 \pm 0.13$ and the density $1.031 \pm 0.002 \mathrm{~g} / \mathrm{cm} 3$, according to the values reported in the literature. The liquid soap developed had organoleptic characteristics, $\mathrm{pH}$ and proper bulk density. Furthermore, further research should be done for product improvements and effectiveness reviews.

Keywords: Agroindustry; Bioactives; Extracts; Waste; Sapindaceae.

\section{Resumen}

Talisia esculenta (A.ST.-HIL.) Radlk, que pertenece a la familia Sapindaceae y se consume ampliamente in natura, principalmente en el noreste de Brasil. El presente estudio tuvo como objetivo desarrollar un jabón líquido con extracto de la cáscara de la pitomba, que es un residuo sólido que generalmente se descarta sin uso. Las cáscaras se trituraron y se almacenaron en botellas de plástico cerradas en un congelador hasta su uso. Posteriormente, se prepararon los extractos hidroalcohólicos y acuosos. Después de este proceso, los extractos fueron analizados por su perfil fitoquímico (saponinas, fenoles y taninos y flavonoles, flavonas, flavonoles y xantonas). A continuación, se desarrolló el jabón líquido. Poco después, se analizaron los parámetros organolépticos, el pH y la densidad aparente. Se encontró que el jabón mostraba una ligera diferencia en el aspecto visual a temperatura ambiente y en el refrigerador y el color a temperatura ambiente, lo que puede estar asociado con la presencia de compuestos bioactivos. El pH fue de $5.44 \pm 0.13$ y la densidad de $1.031 \pm 0.002 \mathrm{~g} / \mathrm{cm} 3$, según los valores reportados en la literatura. El jabón líquido desarrollado tenía características organolépticas, $\mathrm{pH}$ y densidad aparente adecuada. Además, se deben realizar más investigaciones para mejorar los productos y revisar la efectividad.

Palabras clave: Agroindustria; Bioactivos; Extractos; Desperdicio; Sapindaceae.

\section{Introdução}

A Talisia esculenta (A.ST.-HIL.) Radlk, pertencente à família Sapindaceae, é popularmente denominada pitombeira, pitomba, pitomba-da-mata, pitomba-de-macaco, olho-de-boi ou pitombarana. É nativa da Amazônia e amplamente cultivada em diversos estados brasileiros, como no Pará, Maranhão, Ceará, Bahia, Minas Gerais, Rio de Janeiro e Paraná, sendo muito consumida in natura principalmente no Nordeste do Brasil (Guarim Neto, Santana \& Silva, 2003; Sales Junior, 2019).

Os extratos brutos e flavonoides obtidos de plantas pertencentes à família da Sapindaceae vêm sendo pesquisados por apresentarem atividades antidiabéticas, antivirais e anticancerígenas (Neri-Numa et al., 2014). Na folha, na casca do fruto, na semente e na polpa da pitomba foram encontrados compostos fenólicos e flavonoides, sendo as concentrações de acordo com o farmacógeno e o solvente utilizado (Souza, 2016; Fraga, 2018; Sales Júnior, 2019).

A casca do fruto da pitomba apresenta elevado teor de minerais, como potássio e valor semelhante de proteínas quando comparado à polpa, demonstrando potencial valor nutritivo e alto rendimento em relação ao fruto (Fraga, 2018). De acordo com Souza (2016) a casca do fruto apresenta a maior quantidade de polifenóis quando comparado a outras partes da pitomba. Entretanto, as únicas partes aproveitadas são a polpa e as sementes para o consumo e a preparação de chás, respectivamente, sendo a casca descartada (Neto, Santana \& Silva, 2000; Guarim Neto et al., 2003). Segundo o estudo realizado pela Organização das Nações Unidas para a Alimentação e Agricultura (FAO) cerca de 1,3 bilhões de toneladas de alimentos são desperdiçados por ano e causam impactos negativos na economia, além de prejuízos nos recursos naturais que são de suma necessidade para a humanidade (Santos, Panizzon, Cenci, Grabowski \& Jahno, 2020).

Na agroindústria é comum o descarte de cascas na produção de alimentos, o que gera grande quantidade de lixo orgânico e, consequentemente, problemas ambientais e financeiros. Destaca-se que no setor alimentício cerca de $40 \%$ de resíduos agroindustriais são provenientes do processo de produção, sendo caracterizado como um dos fatores negativos para a indústria (Souza et al., 2016). Diaz, Blandino e Caro (2018) reportam que a produção de resíduos é considerada um problema mundial, sendo ocasionado principalmente por indústrias das áreas agrícola, sanitária e de sólidos urbanos. Em contrapartida, resíduos como a casca tem potencial finalidade para alimentos, extração de compostos bioativos, óleos essenciais, vitaminas e minerais, 
sendo úteis na indústria alimentícia, farmacêutica e cosmética (Sousa et al., 2020). Tal fato incentiva o desenvolvimento de produtos na área da biotecnologia sustentável com o objetivo de reduzir os desperdícios e consequentemente disponibilizar produtos de baixo custo e com potencial terapêutico (Schenberg, 2010).

Nesta perspectiva, e considerando que há necessidade do desenvolvimento de produtos de baixo custo e que apresente benefícios para a sociedade, este estudo teve como objetivo desenvolver um sabonete líquido com extrato da casca do fruto da pitomba que é um resíduo sólido que geralmente é descartado sem uso.

\section{Metodologia}

A pesquisa foi realizada no laboratório de desenvolvimento de produtos farmacêuticos da Universidade de Fortaleza (UNIFOR) e consistiu-se nas etapas de obtenção da casca de pitomba, rendimento, preparo dos extratos, análise fitoquímica, desenvolvimento do sabonete líquido, avaliação dos parâmetros organolépticos, determinação do pH e densidade aparente. O trabalho foi cadastrado no Sistema Nacional de Gestão do Patrimônio Genético e do Conhecimento Tradicional Associado (SisGen) sob autorização A8CB494.

\subsection{Obtenção da casca de pitomba}

Foram obtidos $4 \mathrm{~kg}$ de pitombas no município de Eusébio, Ceará, Brasil ( $3^{\circ} 53^{\prime} 24^{\prime \prime}$ latitude sul e $38^{\circ} 27^{\prime}$ '02" longitude oeste) em 25 de janeiro de 2021. Realizou-se a higienização dos frutos com hipoclorito de sódio na concentração de $2 \%$ e procedeu-se com a separação manual da polpa, semente e a casca do fruto. Posteriormente, parte das cascas foram trituradas em liquidificador (Philips Walita, São Paulo, Brasil) e em seguida armazenadas em frascos de plástico fechado em congelador ($\left.6{ }^{\circ} \mathrm{C}\right)$ até o uso.

\subsection{Rendimento}

O rendimento da casca do fruto foi determinado segundo a equação a seguir:

$$
\text { Rendimento }(\%)=\frac{\text { peso da casca }}{\text { peso do fruto inteiro }} \times 100
$$

\subsection{Preparo dos extratos: hidroalcoólico e aquoso}

Para a obtenção do extrato foi utilizado a metodologia descrita por Guedes (2018) com modificações. Inicialmente, foram preparados extrato hidroalcoólico a 20\% (2 g da casca e $10 \mathrm{~mL}$ de solução hidroalcoólica a 70\%) para análise de flavonóis, flavonas, flavononóis e xantonas e extrato aquoso a 20\% (40 g da casca pulverizada e $200 \mathrm{~mL}$ de água destilada) para saponinas, taninos e fenóis e para testagem de formulação, ambos extratos por decocção em chapa aquecedora (Plataforma Quimis, São Paulo, Brasil) por 10 min após o início da ebulição (Matos, 2009). Após a análise fitoquímica, foi adicionado $0,2 \%$ (m/v) de nipagin (metilparabeno) com a função de conservante no extrato aquoso a 20\% (Oliveira, 2019). Foi realizada a filtração do extrato em papel filtro após sete dias de repouso em geladeira (com o objetivo que as substâncias - presente no extrato sedimentem e reduza a precipitação no produto). Posteriormente o extrato foi utilizado no desenvolvimento do sabonete líquido.

\subsection{Análise fitoquímica}

\subsubsection{Saponinas}

Para a análise de saponinas foi transferido $3 \mathrm{~mL}$ do extrato aquoso a 20\% para um tubo de ensaio e agitado manualmente por 15 seg. Foi observado se houve formação de espuma por pelo menos 15 min (Matos, 2009). 


\subsubsection{Fenóis e taninos}

Foi transferido $10 \mathrm{~mL}$ do extrato aquoso a 20\% para um funil de separação e adicionou-se $2 \mathrm{~mL}$ de éter etílico (99,5\%). Em seguida, realizou-se agitação manualmente e coletou-se a fase etérea para a realização do teste para fenóis e a aquosa para taninos. Na fase etérea, foi adicionado $0,5 \mathrm{~mL}$ de etanol e duas gotas de solução de cloreto férrico $10 \%(\mathrm{~m} / \mathrm{v})$ em cada tubo identificado. Foi observado se houve formação de coloração verde a azul escura para resultado positivo. Para taninos foi retirado $1 \mathrm{~mL}$ da fase aquosa e transferido para um tubo de ensaio previamente identificado, em seguida foram adicionadas duas gotas de solução de cloreto férrico a $10 \%$ (m/v). Foi observado se houve a formação de coloração ou precipitado escuro para o resultado ser positivo (Matos, 2009).

\subsubsection{Flavonóis, flavonas, flavononóis e xantonas}

Foi transferido $2 \mathrm{~mL}$ de extrato hidroalcoólico a $20 \%$ para um tubo de ensaio e adicionado $0,5 \mathrm{~mL}$ de $\mathrm{HCl}$ (37\%), e 1,0 $\mathrm{cm}$ de fita de magnésio. Foi aguardado o fim da efervescência (Reação de cianidina). Foi observado se houve aparecimento ou intensificação de cor vermelha, que indica a presença de flavonóis, flavonas, flavononóis e/ou xantonas, livres ou seus heterosídeos para resultado positivo (Matos, 2009).

\subsection{Formulação do sabonete líquido}

Para a formulação, utilizou-se a metodologia reportada no Formulário de Fitoterápicos da Farmacopeia Brasileira (2011) com modificações. Em $20 \mathrm{~mL}$ do extrato aquoso a $20 \%$ em temperatura ambiente (entre 20 e $25^{\circ} \mathrm{C}$ ) adicionou-se $40 \mathrm{~mL}$ de lauril éter sulfato de sódio e $4 \mathrm{~mL}$ de dietanolamina de ácido graxo de coco 90 sob lenta agitação. Posteriormente foi adicionado propilenoglicol e glicerina e realizou-se a homogeneização. Por fim, completou-se o volume com água destilada até $100 \mathrm{~mL}$. Utilizou-se ácido cítrico a 20\% para ajuste de pH, conforme exposto na Tabela 1.

Tabela 1. Formulação do sabonete líquido para $100 \mathrm{~mL}$.

\begin{tabular}{lcc}
\hline \multicolumn{1}{c}{ Componente } & Concentração (\%) & Função \\
\hline Lauril éter sulfato de sódio & $40 \%$ & Detergente \\
Extrato aquoso a 20\% & $20 \%$ & Ativo \\
Propilenoglicol & $4 \%$ & Umectante \\
Glicerina & $4 \%$ & Umectante \\
Dietanolamina de ácido graxo do coco 90 & $4 \%$ & Estabilizante de espuma \\
Ácido cítrico 20\% & q. s. p. & Ajuste de pH \\
Água destilada & q. s. p 100\% & Veículo \\
\hline
\end{tabular}

Fonte: Autores.

\subsection{Parâmetros organolépticos}

As características organolépticas foram determinadas macroscopicamente de acordo com o Guia de Estabilidade de Produtos Cosméticos (Brasil, 2004). Nos dias $0,15^{\circ}, 30^{\circ}, 60^{\circ}$ e $90^{\circ}$ em temperatura ambiente (entre 20 e $25^{\circ} \mathrm{C}$ ) e geladeira (entre 4 e $\left.5^{\circ} \mathrm{C}\right)$.

\subsubsection{Aspecto visual}

Foi observado visualmente se o produto se manteve fluído e homogêneo, sendo classificado como: normal (N), sem alteração; levemente separado (LS), levemente precipitado (LP) ou levemente turvo (LT); separado, precipitado ou turvo (P) (Brasil, 2004). 


\subsubsection{Cor}

A avaliação da cor foi realizada pelo método visual, observando a cor levemente amarelo e as amostras foram classificadas como normal (N), sem alteração; levemente modificada (LM); modificada (M) ou intensamente modificada (IM) (Brasil, 2004).

\subsubsection{Odor}

O odor foi comparado diretamente pelo olfato, observando o odor característico da casca do fruto e as amostras qualificadas em: normal (N), sem alteração; levemente modificada (LM); modificada (M); intensamente modificada (IM) (Brasil, 2004).

\section{$2.7 \mathrm{pH}$}

Para aferir o pH do sabonete líquido utilizou-se o pHmetro (R-TEC-7-MP, São Paulo, Brasil) previamente calibrado com as soluções tampões pH 4,0 e 7,0 (Brasil, 2004). Verificou-se o pH nos dias $0,15^{\circ}, 30^{\circ}, 60^{\circ}$ e $90^{\circ} \mathrm{em}$ temperatura ambiente (entre 20 e $25^{\circ} \mathrm{C}$ ) e geladeira (entre 4 e $5^{\circ} \mathrm{C}$ ).

\subsection{Determinação da densidade aparente}

A densidade foi realizada em picnômetro de vidro de $25 \mathrm{~mL}$ para a formulação, no qual foram pesados os picnômetros vazios e anotado seu peso (M0). Em seguida, o volume do picnômetro foi preenchido com água purificada e pesada (M1). Depois de seco cuidadosamente, foi completamente preenchido com a amostra e pesado (M2) em balança analítica (Tecnal, São Paulo, Brasil) (Brasil, 2007). A densidade foi verificada em temperatura ambiente (entre 20 e $25^{\circ} \mathrm{C}$ ) no 15 o dia. Com os valores obtidos a densidade foi calculada de acordo com a equação abaixo:

$$
D=\frac{\mathrm{M} 2-\mathrm{M} 0}{\mathrm{M} 1-\mathrm{M} 0}
$$

Onde:

$\mathrm{D}=$ Densidade $\left(\mathrm{g} / \mathrm{cm}^{3}\right)$

M0 = Massa do picnômetro vazio, em gramas;

M1 = Massa do picnômetro com água purificada, em gramas;

M2 = Massa do picnômetro com a amostra, em gramas.

\subsection{Análise de dados}

As análises foram realizadas em triplicatas. Os resultados das análises de rendimento $(\mathrm{n}=20), \mathrm{pH}$ e densidade aparente $(\mathrm{n}=3)$ foram expressos como média \pm desvio padrão. Utilizou-se o programa Statistica 10.0 (StatSoft, Inc).

\section{Resultados e Discussão}

O valor do rendimento da casca dos frutos inteiros de pitomba foi de 33,25\% conforme apresentado na tabela 2, resultado inferior ao relatado por Fraga (2018) com valor de 44,60\%. Tem-se reportado rendimentos menores para fontes da família Sapindaceae, como apresentado por Queiroz, Abreu e Oliveira (2012) que relataram valor de 27,07\% para a casca da lichia (Litchi chinensis Sonn).

É importante ressaltar que no protocolo de extração de frutos, as principais perdas acontecem principalmente na remoção da casca e polpa, homogeneização e/ou trituração (Oliveira, Fuchs, Gonçalves \& Moreira, 2018). 
Research, Society and Development, v. 10, n. 15, e325101522791, 2021

(CC BY 4.0) | ISSN 2525-3409 | DOI: http://dx.doi.org/10.33448/rsd-v10i15.22791

Tabela 2. Proporção de frutos inteiros de pitomba (Talisia esculenta Radlk) e casca.

\begin{tabular}{ccc}
\hline Frações & Massa (g) & Proporção (\%) \\
\hline Fruto inteiro & $7,97 \pm 1,23$ & 100 \\
Casca & $2,65 \pm 0,46$ & 33,25 \\
\hline
\end{tabular}

Resultados expressos em média $(\mathrm{n}=20) \pm$ desvio padrão.

Fonte: Resultados da pesquisa (2021).

Os resultados obtidos na análise fitoquímica estão expostos na Figura 1. No teste qualitativo do extrato aquoso a $20 \%$ houve presença persistente de espuma por mais de $15 \mathrm{~min}$, sendo positivo para saponinas. Para fenóis o resultado foi negativo, pois não houve mudança de cor. Contudo, foi positivo para taninos e flavonóis, flavonas, flavononóis e xantonas, apresentando mudança de coloração.

Dantas et al. (2020) analisou os caroços da pitomba e reportou a presença de fenóis, taninos condensados, flavonóides, flavonas, flavononóis e xantonas. Entretanto, não houve presença de saponinas. Diferente ao reportado neste estudo que se observou a presença de saponina na casca da pitomba. Destaca-se que as principais atividades encontradas nas saponinas são anti-inflamatórias, antifúngicas, antimicrobiana, antivirais e hemolíticas (Fernandes et al., 2019).

Fraga (2018) reporta que o extrato etanólico da casca $(5,9 \pm 0,07 \mu \mathrm{g}$ GAE/mg de extrato) apresentou maiores

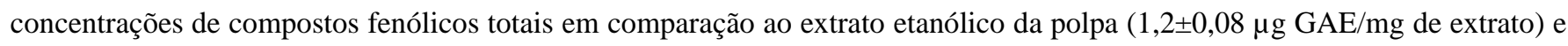
flavonoides (19,12 $\pm 0,04 \mu \mathrm{g} \mathrm{CE} / \mathrm{mg}$ de extrato), quantidade superior ao da polpa $(0,96 \pm 0,01 \mu \mathrm{g} \mathrm{CE} / \mathrm{mg}$ de extrato).

Souza (2016) destaca também que o extrato metanólico da casca da pitomba possui maiores concentraç̃es de compostos fenólicos totais, sendo, $0,363 \pm 0,04$ para casca, $0,057 \pm 0,03$ para semente e $0,038 \pm 0,05$ para polpa, em mg GAE/100g extrato seco.

Figura 1. (A) positivo para saponinas; (B) negativo para fenóis; (C) positivo para taninos; (D) positivo para flavonóis, flavonas, flavononóis e xantonas.
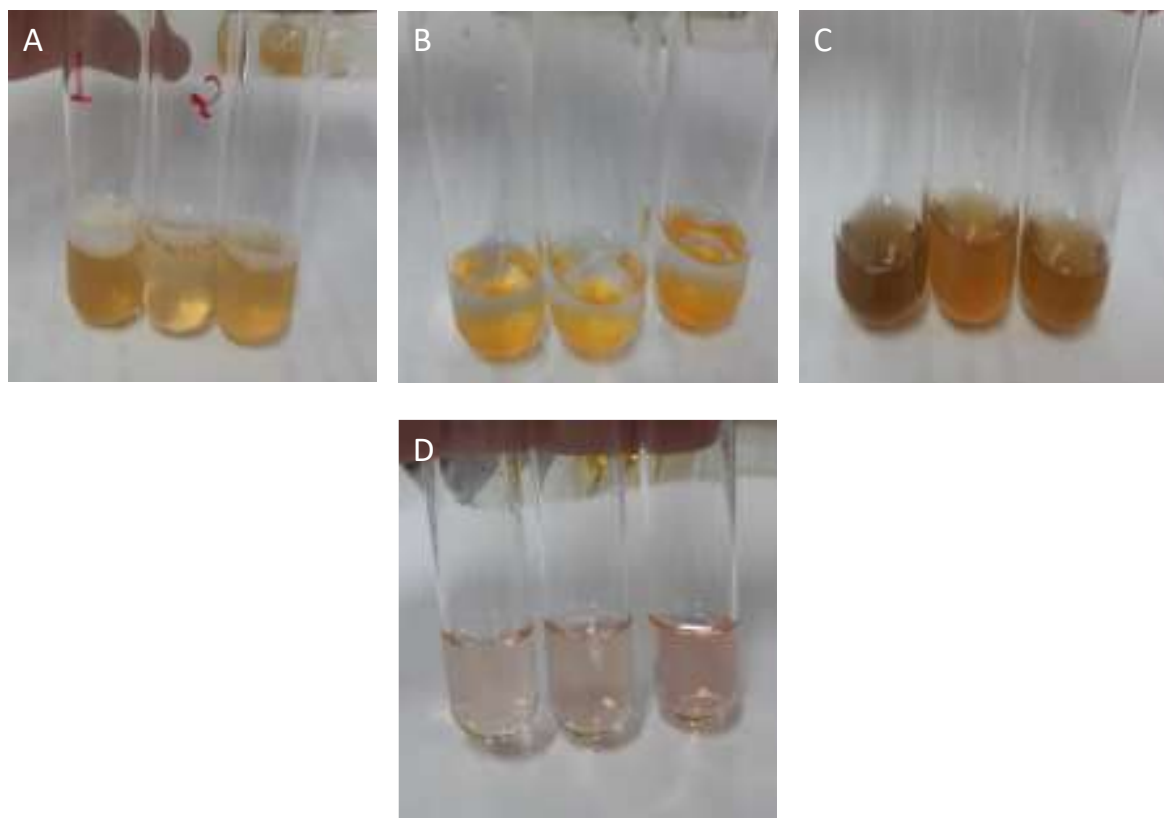

Fonte: Resultados da pesquisa (2021). 
Os resultados referentes aos parâmetros organolépticos e pH do produto desenvolvido estão presentes nas tabelas 3 e 4 . Observou-se que não houve diferenças significativas nas características organolépticas e pH do produto, que se manteve estável durante os dias de análise.

Foi observada discreta diferença no aspecto visual em temperatura ambiente e geladeira e cor em temperatura ambiente, ambos a partir do $15^{\circ}$ dia, que passou a ser levemente precipitado e levemente modificada, respectivamente. Tal diferença pode ser pelo o fato que o extrato apresenta compostos fenólicos, como taninos, que são muito reativos, podendo ser oxidados por metais ou enzimas vegetais, além de possuir outros compostos fotossensíveis. Dessa maneira, acarreta escurecimento de soluções e precipitação de proteínas durante seu processamento (Monteiro, Albuquerque, Araújo \& Amorim, 2005; Rocha et al., 2011). Vale salientar que para garantir as características adequadas de conservação e armazenamento, o ideal é armazenar o produto em frasco âmbar com o intuito de proteger os ativos da luz.

Ressalta-se que os parâmetros organolépticos validam as características de aceitação do produto pelo consumidor, além de fazer parte da garantia de qualidade do produto (Brasil, 2004). Um dos indícios de instabilidade físico-química de formulações são modificações nas características organolépticas relacionados a cor e odor (Souza \& Nóbrega, 2018). Por exemplo, compostos bioativos podem ser instáveis quando expostos a luz e oxigênio, podendo degradá-los (Neves et al., 2021). Ou seja, são parâmetros de extrema importância para assegurar a qualidade, eficácia e segurança do produto (Lopes, Castilho \& Castro 2019).

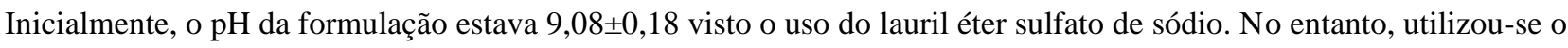
ácido cítrico a 20\% para ajustar o $\mathrm{pH}$ do produto para 5,44 $\pm 0,13$ considerado o ideal para o uso na pele humana, na qual apresenta pH entre 4,5 a 6,0 (Lopes et al, 2018). No estudo de Soares (2014), os valores de pH de sabonetes líquidos variaram entre 5,87 a 6,35, sendo o primeiro valor o sabonete com óleo de buriti (Mauritia flexuosa L.) considerado o melhor para uso na pele. Destaca-se que produtos sólidos, como por exemplo sabonete em barra, não são recomendados por serem mais abrasivos e normalmente terem pH alcalino que reduz atividade antimicrobiana (Bezerra, Souza, Carmo, \& Luis 2016).

Tabela 3. Parâmetros organolépticos e pH do sabonete líquido em temperatura ambiente (entre 20 e $25^{\circ} \mathrm{C}$ ).

\begin{tabular}{cccccc}
\hline Ambiente & $\mathbf{0}$ & $\mathbf{1 5}^{\mathbf{0}}$ & $\mathbf{3 0}^{\mathbf{0}}$ & $\mathbf{6 0}^{\mathbf{0}}$ & $\mathbf{9 0}^{\mathbf{0}}$ \\
\hline Aspecto visual & $\mathrm{N}$ & $\mathrm{LP}$ & $\mathrm{LP}$ & $\mathrm{LP}$ & $\mathrm{LP}$ \\
Cor & $\mathrm{N}$ & $\mathrm{LM}$ & $\mathrm{LM}$ & $\mathrm{LM}$ & LM \\
Odor & $\mathrm{N}$ & $\mathrm{N}$ & $\mathrm{N}$ & $\mathrm{N}$ & $\mathrm{N}$ \\
$\mathrm{pH}$ & $5,44 \pm 0,13$ & $5,37 \pm 0,03$ & $5,73 \pm 0,17$ & $5,74 \pm 0,01$ & $5,45 \pm 0,17$ \\
\hline
\end{tabular}

Legendas: N - normal; LP - levemente precipitado; LM - levemente modificada. Fonte: Resultados da pesquisa, 2021.

Tabela 4. Parâmetros organolépticos e pH do sabonete líquido em geladeira (entre 4 e $5{ }^{\circ} \mathrm{C}$ ).

\begin{tabular}{cccccc}
\hline Geladeira & $\mathbf{0}$ & $\mathbf{1 5}^{\mathbf{0}}$ & $\mathbf{3 0}^{\mathbf{0}}$ & $\mathbf{6 0}^{\mathbf{0}}$ & $\mathbf{9 0}^{\mathbf{0}}$ \\
\hline Aspecto visual & $\mathrm{N}$ & $\mathrm{LP}$ & $\mathrm{LP}$ & $\mathrm{LP}$ & $\mathrm{LP}$ \\
Cor & $\mathrm{N}$ & $\mathrm{N}$ & $\mathrm{N}$ & $\mathrm{N}$ & $\mathrm{N}$ \\
Odor & $\mathrm{N}$ & $\mathrm{N}$ & $\mathrm{N}$ & $\mathrm{N}$ & $\mathrm{N}$ \\
$\mathrm{pH}$ & $5,44 \pm 0,13$ & $5,35 \pm 0,02$ & $5,72 \pm 0,26$ & $5,58 \pm 0,19$ & $5,43 \pm 0,16$ \\
\hline
\end{tabular}

Legendas: $\mathrm{N}$ - normal; LP - levemente precipitado.

Fonte: Resultados da pesquisa, 2021.

O valor obtido da densidade aparente foi de 1,031 $\pm 0,002 \mathrm{~g} / \mathrm{cm}^{3}$. No estudo realizado por Higioka e Barzotto (2013), os valores de densidade do sabonete líquido desenvolvido foram de 1,002 e 1,003 g/ $\mathrm{cm}^{3}$. Conforme o estudo de Soares (2014), a densidade relativa do sabonete líquido comercial analisado foi $1,022 \pm 0,045 \mathrm{~g} / \mathrm{cm}^{3}$, com óleo de Baru (Dipteryx alata Vog)

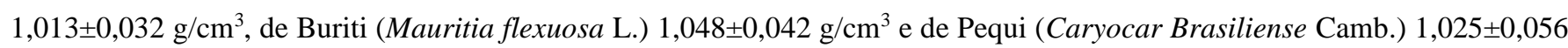


$\mathrm{g} / \mathrm{cm}^{3}$, representando uma média de $1,028 \mathrm{~g} / \mathrm{cm}^{3}$. Os presentes estudos reportados se assemelham com o valor obtido neste estudo. É importante verificar a densidade aparente do sabonete líquido, visto que é uma análise que permite monitorar a qualidade e as características do produto durante o seu prazo de validade. Em líquidos e semissólidos, esse parâmetro pode demonstrar incorporação de ar ou perda de compostos voláteis (Brasil, 2004).

Cabe mencionar que a elaboração de produtos de baixo-custo advindos de matérias-primas que seriam descartadas ajuda a impulsionar o desenvolvimento econômico e regional. No entanto, a exploração inadequada da flora local pode ocasionar à perda de espécies nativas e ocasionar sérios problemas (Schenberg, 2010; Souza et al., 2016; Diaz, Blandino \& Caro 2018).

Até o momento não se tem na literatura trabalhos que utilizem as cascas da pitomba para o desenvolvimento de sabonete líquido, o que garante o aspecto inovador do produto desenvolvido e acrescenta mais alternativas de aproveitamento deste resíduo que seria descartado. A compreensão sobre os constituintes químicos presente na casca do fruto, in natura, permite melhores benefícios.

\section{Conclusão}

O sabonete líquido desenvolvido apresentou características organolépticas, $\mathrm{pH}$ e densidade aparente em conformidade com valores reportados na literatura para sabonetes líquidos comerciais e desenvolvidos a partir de extratos vegetais. Dessa forma, é demonstrado que as cascas da pitomba, subproduto de descarte, pode ser utilizado no desenvolvimento de produtos farmacêuticos que apresentam características adequadas de uso. Ademais, pesquisas posteriores deverão ser feitas para melhorias do produto desenvolvido e realização das análises, in vitro e in vivo, para avaliar a atividade antimicrobiana e a eficácia do sabonete.

\section{Agradecimentos}

Agradecemos à Fundação Cearense de Apoio ao Desenvolvimento Científico e Tecnológico (FUNCAP) e à Universidade de Fortaleza pelo apoio financeiro e de infraestrutura.

\section{Referências}

Bezerra, P. X., Souza, J. B. P., Carmo, E. S., \& Luis, J. A. S. (2016). Avaliação da rotulagem e parâmetros de qualidade de sabonetes íntimos. Revista Brasileira de Ciências da Saúde, 20 (1), 51-60. 10.4034/RBCS.2016.20.01.08

Brasil. (2007). Agência Nacional de Vigilância Sanitária. Guia de controle de qualidade de produtos cosméticos. Anvisa. https://www.gov.br/anvisa/ptbr/centraisdeconteudo/publicacoes/cosmeticos/manuais-e-guias/guia-de-controle-de-qualidade-de-produtos-cosmeticos.pdf/view

Brasil. (2004). Ministério da Saúde. Agência Nacional de Vigilância Sanitária. Guia de estabilidade de produtos cosméticos. https://www.gov.br/anvisa/ptbr/centraisdeconteudo/publicacoes/cosmeticos/manuais-e-guias/guia-de-estabilidade-de-cosmeticos.pdf/view

Brasil. (2011). Agência Nacional de Vigilância Sanitária. Formulário de Fitoterápicos da Farmacopéia Brasileira/Agência Nacional de Vigilância Sanitária. Anvisa. https://www.gov.br/anvisa/pt-br/assuntos/farmacopeia/formulario-fitoterapico

Dantas, L. V. B., Lopes, F. F. S., Alves, D. R., Frota, L. S., Cardoso, A. L. H., \& Morais, S. M. (2020). Avaliação Fitoquímica, quantificação de Fenóis e Flavonóides totais, Atividade antioxidante e antiacetilcolinesterase do extrato etanólico da Talísia esculenta (Pitomba). Brazilian Journal of Development, 6(8), 60597-60602. 10.34117/bjdv6n8-467

Diaz, A. B.; Blandino, A., \& Caro, I. (2018). Value added products from fermentation of sugars derived from agro-food residues. Trends in Food Science and Technology, 71, 52-64. 10.1016/j.tifs.2017.10.016

Fernandes, B. F., Gonçalves, H. R., Guimarães, M. R., Alves, A. A., \& Bieski, I. G. C. (2019). Estudo etnofarmacológico das plantas medicinais com presença de saponinas e sua importância medicinal. Revista da Saúde da AJES, Juína/MT, 5 (9), 6-22.

Fraga, L. N. (2018). Compostos bioativos, capacidade antioxidante e citotoxicidade da casca e polpa da pitomba (Talisia esculenta (ST. HIL.) RALDK). Dissertação de mestrado, Universidade Federal de Sergipe, São Cristóvão, SE, Brasil. https://ri.ufs.br/handle/riufs/8853

Guarim Neto, G., Santana, S. R., \& Silva, J. V. B. (2003) Repertório botânico da pitombeira (Talisia esculenta (St.-Hil.) Radlk. - Sapindaceae). Acta Amazonica, 33 (2), 237-242. 10.1590/1809-4392200332242 
Higioka, A. S., \& Barzotto, I. L. M. (2013). Desenvolvimento e controle físico-químico de sabonete líquido com digluconato de clorexidina. Rev. Ciênc. Farm. Básica Apl., 34 (4), 537-543.

Lopes, E. M. X., Castilho, F. L. M., \& Castro, V. C. F. (2019). Análises de qualidade de xampus de cetoconazol 2\% manipulados em farmácias magistrais de Porto Velho, RO, Brasil. Infarma-Ciências Farmacêuticas, 31(3), 155-162. 10.14450/2318-9312.v31.e3.a2019.pp155-162

Lopes, A. C., Moraes, A., Oliveira, A. F. R., Guimarães, B. P., Oliveira, C. F., Betim, F. C. M., Martinez, A. L., \& Bobek, V. B. (2018). Análise físico-química comparativa de sabonetes líquidos. Visão Acadêmica, 19 (2), 84-89. 10.5380/acd.v19i2.58026

Matos, F. J. A. (2009). Introdução à fitoquímica experimental. (3. Ed). Fortaleza-CE: Edições Universidade Federal do Ceará (UFC).

Monteiro, J. M., Albuquerque, U. P., Araújo, E. L., \& Amorim, E. L. C. (2005). Taninos: uma abordagem da química à ecologia. Quim. Nova, 28 (5), 892-896. 10.1590/S0100-40422005000500029

Neri-Numa, I. A., Carvalho-Silva, L. B., Ferreira, J. E. M., Machado, A. R. T., Malta, L. G., Ruiz, A. L. T. G., Carvalho, J. E., \& Pastore, G. M. (2014). Preliminary evaluation of antioxidant, antiproliferative antimutagenic activities of pitomba (Talisia esculenta). Lwt - Food Science and Technology, 59 (2), 1233-1238. 10.1016/j.1wt.2014.06.034

Neto, G. G.; Santana, S. R.; \& Silva, J. V. B. (2000). Notas etnonobotânicas de espécies de Sapindaceae Jussieu. Acta Botânica Brasilica, 14 (3), $327-334$. 10.1590/S0102-33062000000300009

Neves, M. T., Santos, F. R., Gonçalves, D. J. R., Fernandes, J. G., Justino, H. F. M., Leite Júnior, B. R. C. \& Vieira, E. N. R. (2021). Use of liposome technology in the encapsulation of bioactive compounds - Review. The Journal of Engineering and Exact Sciences - Jcec, 7 (4), 1-20. 10.18540/jcecv17iss4pp13295-01$20 \mathrm{e}$

Oliveira, E. S., Fuchs, M. C. L., Gonçalves, N. G. G., \& Moreira, R. A. (2018). Extração do amido de fruta-pão (Artocarpus altilis) variedade apyrena [Resumo]. In Encontros Científicos da Universidade de Fortaleza (UNIFOR) (Eds.) Resumos de comunicações científicas, XXIV - Encontro de Iniciação à Pesquisa. (p. 16). Fortaleza-CE, Brasil.

Oliveira, W. C. (2019). Avaliação da eficácia conservante em gel de babosa para a farmácia escola manoel casado de almeida. Monografia (Especialização), Universidade Federal de Campina Grande, Cuité, Pb, Brasil. http://dspace.sti.ufcg.edu.br:8080/xmlui/handle/riufcg/8342

Queiroz, E. R., Abreu, C. M. P., \& Oliveira, K. S. (2012). Constituintes químicos das frações de lichia in natura e submetidas à secagem: potencial nutricional dos subprodutos. Revista Brasileira de Fruticultura, 34 (4), 1174-1179. 10.1590/S0100-29452012000400026

Rocha, W. S., Lopes, R. M., Silva, D. B., Vieira, R. F., Silva, J. P., \& Agostini-Costa, T. S. (2011). Compostos fenólicos totais e taninos condensados em frutas nativas do cerrado. Rev. Bras. Frutic. 33 (4), 1215-1221. 10.1590/S0100-29452011000400021

Sales Junior, J. H. (2019). Avaliação da atividade antimicrobiana do extrato hidroetanólico de Talisia esculenta Radlk. Dissertação (Programa de Pós-Graduação em Saúde do Adulto e da Criança/CCBS). Universidade Federal do Maranhão, São Luís, MA, Brasil. https://tedebc.ufma.br/jspui/handle/tede/3066

Santos K. L., Panizzon J., Cenci M. M., Grabowski G., \& Jahno V. D. (2020). Perdas e desperdícios de alimentos: reflexões sobre o atual cenário brasileiro. Braz. J. Food Technol. 23: e2019134. 10.1590/1981-6723.13419

Schenberg, A. C. G. (2010). Biotecnologia e desenvolvimento sustentável. Dossiê Biotecnologia - Estud. av., 24 (70), 7-17. 10.1590/S0103-40142010000300002

Soares, N. R. (2014). Avaliação da atividade antimicrobiana e caracterização físico-química de sabonete líquido à base de óleo de baru, buriti e pequi. Dissertação (Mestrado em Ciência e Tecnologia de Alimentos), Universidade Federal de Goiás, Goiás, GO, Brasil. repositorio.bc.ufg.br/tede/hamdle/tede/3885

Sousa, H. M. S., Santos, A. C., Glêndara, C. D., \& Martins A. S. (2020). Resíduos de frutos: compostos de interesse. In T. Magrini (Ed.), Aproveitamento de resíduos vegetais: potenciais e limitações (pp. 20-38). Palmas, TO: EDUFT.

Souza, A. L. G.; Ferreira, M. C. R.; Corrêa, N. C. F.; \& Santos, O. V. (2020). Aproveitamento dos resíduos de extração de óleo da castanha-do-brasil (Bertholletia excelsa) em produtos alimentícios ricos em proteínas, lipídios e fibras. Revista Pan-Amaz Saúde, 7 (4), 21-30. 10.5123/s2176-62232016000400003

Souza, J. P. B., \& Nóbrega, R. S. (2018). Avaliação de parâmetros físico-químicos e eficácia de formulações comerciais de xampus de cetoconazol. Revista Saúde \& Ciência Online, 7(1) 45-64. 10.35572/rsc.v7i1.80

Souza, M. P. (2016). Caracterização química e avaliação do potencial antioxidante dos frutos mari-mari (Cassia leiandra), pajurá (Couepia bracteosa) e pitomba (Talisia esculenta). Tese (Doutorado em Química), Universidade Federal do Amazonas, Manaus, AM, Brasil. https://tede.ufam.edu.br/handle/tede/5598 